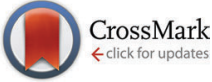

Cite this: Chem. Commun., 2014, 50, 9973

Received 15th May 2014 Accepted 11th July 2014

DOI: $10.1039 / c 4 c c 03723 e$

www.rsc.org/chemcomm

\section{Convergent and divergent two-dimensional coordination networks formed through substrate- activated or quenched alkynyl ligation $\dagger$}

\author{
Jan Čechal, ${ }^{\text {ab }}$ Christopher S. Kley, ${ }^{a}$ Takashi Kumagai, ${ }^{a c}$ Frank Schramm, ${ }^{d}$ \\ Mario Ruben, ${ }^{\text {de }}$ Sebastian Stepanow ${ }^{{ }^{\text {af }}}$ and Klaus Kern ${ }^{\text {ag }}$
}

\begin{abstract}
Metal coordination assemblies of the symmetric bi-functional 4,4'-di-(1,4-buta-1,3-diynyl)-benzoic acid are investigated by scanning tunnelling microscopy on metal surfaces. The formation of long-range ordered, short-range disordered and random phases depends on the competition between the convergent and divergent coordination motifs of the individual functional groups and is crucially influenced by the substrate.
\end{abstract}

Molecular self-assembly at surfaces has become an area of intense research. Its increasing popularity is largely attributed to the real space imaging capability of scanning probe techniques with single molecule resolution. The main interest lies in the understanding of self-assembly phenomena of complex systems with future prospects for molecular electronics, heterogeneous catalysis, and molecular templates among other topics. ${ }^{1}$

A variety of fascinating and complex self-assembled motifs have been observed. These are essentially the result of several competing interactions between the components as well as the chemical coupling of the adsorbates to the supporting surface. ${ }^{1-11}$ In general, convergent assemblies with a regular periodic structure in the thermodynamic limit are sought. Hence, the investigation of model systems can provide fundamental insights into

\footnotetext{
${ }^{a}$ Max Planck Institute for Solid State Research, Heisenbergstrasse 1, 70569 Stuttgart, Germany

${ }^{b}$ CEITEC and Institute of Physical Engineering, Brno University of Technology, Technická 3058/10, 61600 Brno, Czech Republic. E-mail: cechal@fme.vutbr.cz ${ }^{c}$ Department of Physical Chemistry, Fritz-Haber Institute of the Max-Planck Society, Faradayweg 4-6, 14195 Berlin, Germany

${ }^{d}$ Institute of Nanotechnology, Karlsruhe Institute of Technology, D-76344 Eggenstein-Leopoldshafen, Germany

${ }^{e}$ IPCMS-CNRS UMR 7504, Université de Strasbourg, 23 Rue du Loess, F-67034 Strasbourg, France

${ }^{f}$ Department of Materials, ETH Zürich, Hönggerbergring 64, 8093 Zürich, Switzerland. E-mail: sebastian.stepanow@mat.ethz.ch

${ }^{g}$ Institute de Physiques de la Matière Condensée,

Ecole Polytechnique Fédérale de Lausanne, 1015 Lausanne, Switzerland

$\dagger$ Electronic supplementary information (ESI) available: A detailed description of experimental procedures and statistical analysis of Ni-1 binding motifs. See DOI: 10.1039/c4cc03723e
}

the thermodynamics and kinetics of complex multicomponent systems. ${ }^{2,3,12-15}$ Particularly, apart from long-range ordered networks, ${ }^{3,10}$ short-range disordered ${ }^{5}$ and random systems ${ }^{6-9}$ are of interest due to the possibility of direct visualization of basic structural units at the molecular level and their correlation with the molecular geometry.

The employed substrate also plays an important role in the self-assembly process. ${ }^{1}$ It generally confines the adsorbates to the surface plane and puts constraints on the molecular adsorption geometries, which leads to the well-known templating effects. ${ }^{1}$ Furthermore, charge transfer from the substrate can alter the electronic states of the adsorbates and thus their physical properties. ${ }^{16}$

In this communication we show that the activation of the coordination chemistry of an additional alkynyl functional group of the symmetric, bi-functional organic ligand 4,4'-di-(1,4-buta-1,3diynyl)-benzoic acid (1, see Scheme 1 ) leads to a crossover from long-range ordered convergent coordination assemblies to divergent short-range disordered or even random coordination structures with $\mathrm{Fe}, \mathrm{Ni}$ or $\mathrm{Cu}$ adatoms on metal surfaces. The results highlight the crucial role of the substrate in supramolecular self-assembly as it influences the resulting energy landscape of the competing interactions between the distinct functional groups and mediates their selective activation and incorporation in the binding motifs.

Fig. 1 shows ordered coordination network structures of 1 on $\mathrm{Au}(111), \mathrm{Ag}(001)$ and $\mathrm{Cu}(001)$ substrates prepared via thermal evaporation of all components on a clean substrate and imaged using a scanning tunnelling microscope (STM) operated in an ultrahigh vacuum at room and low temperature $(5 \mathrm{~K})$, respectively. $\dagger$ The homoleptic coordination networks display both long and shortrange order regardless of the employed substrate. Simultaneous deposition of $\mathrm{Fe}$ or $\mathrm{Ni}$ together with 1 on $\mathrm{Au}(111)$ at elevated temperatures results in the formation of fully reticulated

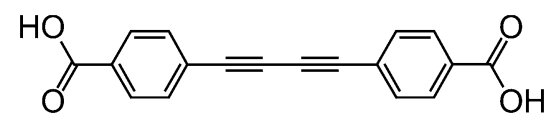

Scheme 1 Chemical structure of the molecule used within this study (1). 


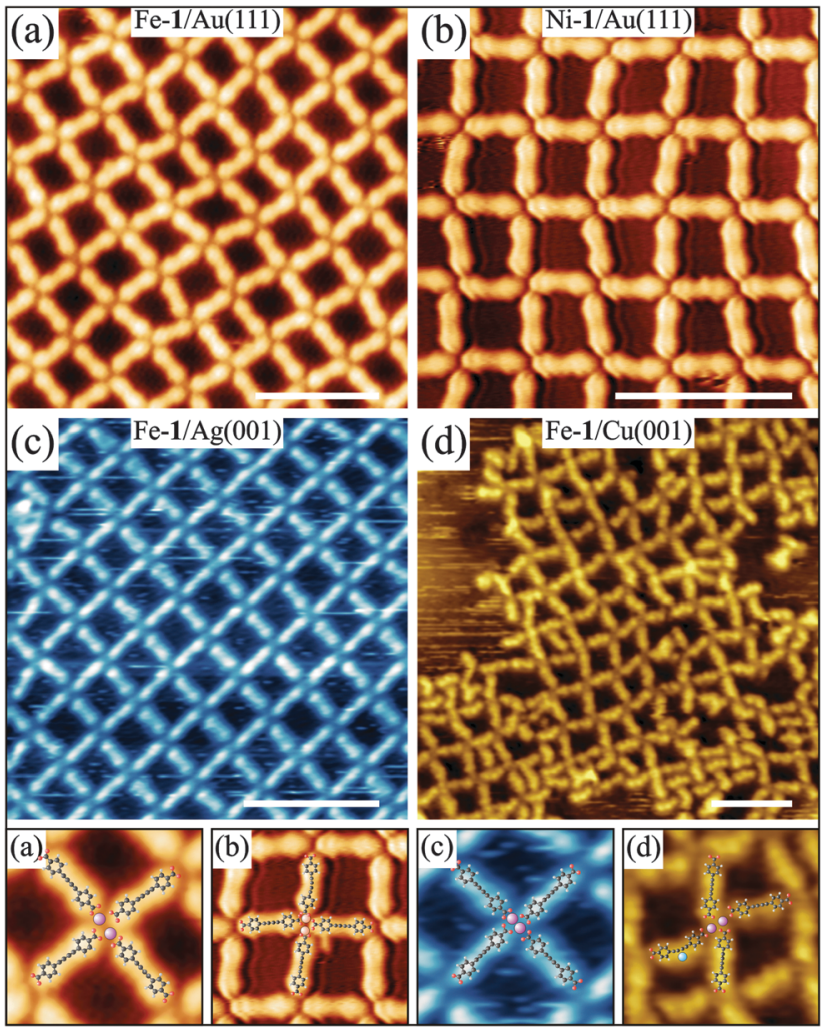

Fig. 1 (a) Fe-1 and (b) Ni-1 fully reticulated network structures on $\mathrm{Au}(111)$ (metal atom: 1 ratio 1:1). The same Fe-1 networks can also be obtained on (c) $\mathrm{Ag}(001)$ and (d) $\mathrm{Cu}(001)$ substrates. Models for each structure are shown in the corresponding bottom panels. Structures were obtained by simultaneous deposition of metal atoms and $\mathbf{1}$ at $450 \mathrm{~K}$ on the substrates. Images acquired at $5 \mathrm{~K}(\mathrm{a}, \mathrm{b})$ and $300 \mathrm{~K}(\mathrm{c}, \mathrm{d})$. Scale bars are $5 \mathrm{~nm}$.

coordination networks based on the $\mathrm{Fe}$ - and $\mathrm{Ni}$-carboxylate coordination motif, respectively (Fig. 1a and b). In particular the Fe-1 networks show a high degree of structural adaptability on the $\mathrm{Au}(111)$ surface, which is also present on $\mathrm{Ag}(001)$, see Fig. 1c. ${ }^{10}$ This is enabled by the conformational flexibility of 1 which arises from partial re-hybridization of the alkynyl moiety in contact with the metal substrate. ${ }^{10}$ On the $\mathrm{Cu}(001)$ surface, the Fe-1 network structure is only poorly formed. Here, Fe-carboxylate coordination still dominates the network assembly but secondary binding motifs involving additional heteroleptic $\mathrm{Cu}$ coordination (see below) between the carboxylate and butadiynyl moiety are also observed (see Fig. 1d). As a consequence a variety of locally distinct binding motifs are recognized; the most prominent ones include incorporation of additional molecules into Fe-1 network cavities, i.e. a side-on binding of $\mathbf{1}$ to the butadiynyl group facilitated by $\mathrm{Cu}$ coordination. This binding multiplicity triggers complexity in the system by inducing structural disorder.

The influence of the finite coupling of $\mathbf{1}$ to the substrate is revealed in the detailed comparison between the Fe-1 networks on the $\mathrm{Au}(111)$ and $\mathrm{Ag}(001)$ substrates ( $c f$. Fig. 1a and c). While convergent coordination assemblies with strict periodic order can be achieved on the square lattice $\mathrm{Ag}(001)$ substrate employing the slow and incremental growth procedure (see Fig. 1c), the network on the threefold symmetric $\mathrm{Au}(111)$ substrate with the preserved herringbone reconstruction necessarily displays a certain degree of disorder on the local scale (Fig. 1a). A more striking difference in the structure formation on the $\mathrm{Au}(111)$ and $\mathrm{Ag}(001)$ substrates is revealed in networks with Fe: 1 ratios differing from $1: 1$. Fig. 2a portrays a Fe-rich $2: 1$ structure on $\mathrm{Au}(111)$ in which 1 and Fe form densely packed one-dimensional chains. The internal ligand flexibility appears to be essential for obtaining this structure as evidenced by the bent shape of several ligands. The ligand's bent conformation also indicates that excess Fe adatoms may participate in coordination interactions with the alkynyl moieties within the molecular rows. On $\mathrm{Ag}(001)$ an increase in the Fe:1 ratio to $2: 1$ leads to the formation of the square network shown in Fig. 1c and excess Fe clusters on the substrate. At the Fe deficient 2:3 ratio a ladder-type structure forms on $\mathrm{Ag}(001)$ as shown in Fig. $2 \mathrm{~b}$ which possesses longrange order only in the direction of the molecular double-rows. These are connected via highly flexible molecular linkers that can adapt to the misalignment of adjacent double-rows. On $\mathrm{Au}(111)$, however, we observe the coexistence of the square network (Fig. 1a) and domains of the non-coordinated molecular phase. Thus, both structures shown in Fig. 2 are unique for the given substrate highlighting its noninnocent role in the self-assembly process while the open square networks shown in Fig. 1 form on all considered substrates.

$\mathrm{Fe}$ and $\mathrm{Ni}$ atoms in the coordination structures grown on the $\mathrm{Au}(111)$ surface exhibit low affinity to coordinate to the central butadiynyl group of $1{ }^{17}$ Hence, apart from their flexibility, the alkynyl groups are not involved in the network formation. Although the change of substrate for the Fe-1 networks can lead to unique substrate dependent structural motifs as shown for the Fe rich and deficient structures on $\mathrm{Au}(111)$ and $\mathrm{Ag}(001)$ surfaces in Fig. 2, the Fe-carboxylate bonding motif prevails throughout all structures. This situation is changed on the $\mathrm{Cu}(001)$ surface, where the butadiyne group becomes coordination active via the involvement of $\mathrm{Cu}$ adatoms. ${ }^{18}$ The coordination bonding is clearly evident by the bent conformation of 1 deposited on the $\mathrm{Cu}(001)$ substrate as shown in Fig. 3. The $\mathrm{Cu}$ adatoms that are imaged as bright spots near the centre of the molecular backbone coordinate to an alkynyl group at the concave side of the bent ligands yielding a structural motif tentatively presented in Fig. 3b. A chiral network pattern is obtained, where the central chiral coordination units (see model in Fig. 3b) are closely packed together possibly via attractive carboxylate

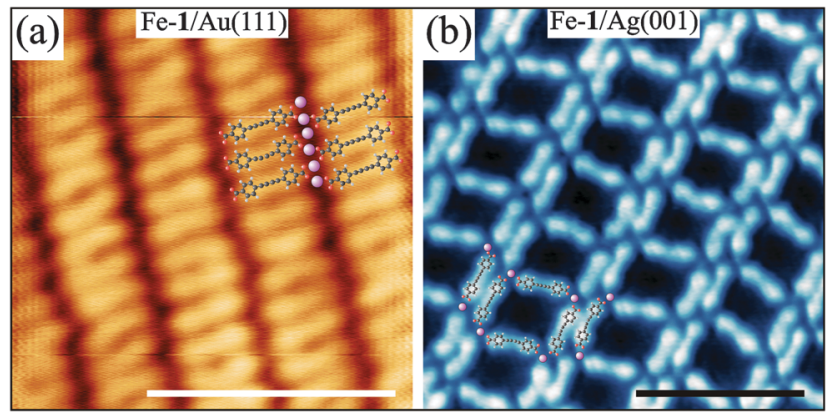

Fig. 2 (a) Fe-rich row phase on $A u(111)$. Fe: 1 ratio $2: 1$. (b) Fe-deficient double-row structure on $\mathrm{Ag}(001)$. Fe:1 ratio: 2:3. Structures were obtained by simultaneous deposition of $\mathrm{Fe}$ and 1 at $450 \mathrm{~K}$. Images acquired at $5 \mathrm{~K}$. Scale bars are $5 \mathrm{~nm}$. 


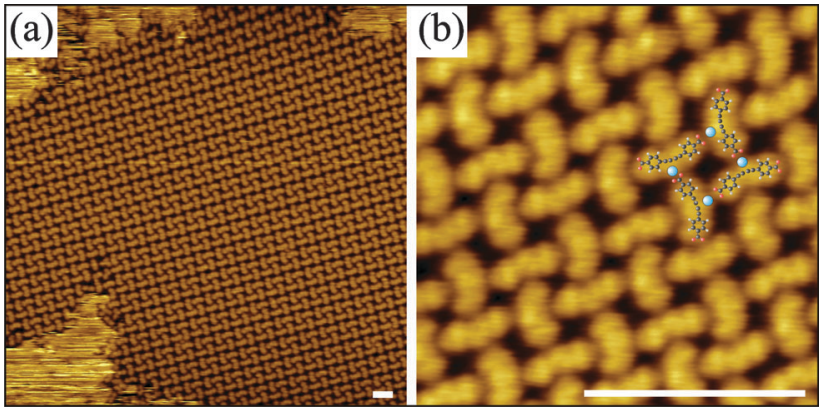

Fig. 3 (a, b) Ordered phase of 1 on $\mathrm{Cu}(001)$. Molecules were deposited at $300 \mathrm{~K}$ and subsequently annealed at $450 \mathrm{~K}$ for $5 \mathrm{~min}$. (b) Blue spheres indicate some $\mathrm{Cu}$ adatoms. Images acquired at $300 \mathrm{~K}$. Scale bars are $5 \mathrm{~nm}$.

hydrogen bonds. Despite the relatively strong conformational change and the emergence of a heteroleptic coordination motif of 1 , which usually lead to disordered structures, ${ }^{14}$ long-range ordered supramolecular domains evolve.

The cooperating $\mathrm{Cu}$-carboxyl and $\mathrm{Cu}$-alkynyl bonding in the $1 / \mathrm{Cu}(001)$ system suggests that the network topology can be steered by the respective activation and strength of the two available coordination modes. Altering the bonding affinity and strength is generally achieved by the choice of the metal coordination centre, but can also be influenced by the ligand-surface interaction and hence the nature of the substrate as demonstrated here. For the first route, $\mathrm{Ni}$ atoms show similarly to $\mathrm{Cu}$ high affinity to form bonds with both carboxyl and alkyne functional groups. ${ }^{19}$ However, on the $\mathrm{Au}(111)$ surface the Ni adatoms only interact with the carboxyl moieties and form regular networks with 1 similar to Fe, see Fig. 1b. In contrast, on the $\mathrm{Ag}(001)$ surface $\mathrm{Ni}$ was reported to coordinate also to the alkyne moiety. ${ }^{18}$ Fig. 4a shows that Ni-1 networks involving coordination also at the alkynyl groups can be grown on $\mathrm{Ag}(001)$. Here, Ni engages in heteroleptic coordination motifs with both carboxylate and alkynyl groups. As a consequence the molecule bends upon alkynyl coordination resulting in strongly distorted L-shaped chiral ligands with lower symmetry than their $\mathrm{Cu}$ analogues, which appear symmetrically bent. The number of possible binding motifs increases with the low-symmetry ligand shapes and possible interaction pathways. ${ }^{11}$ In combination with a

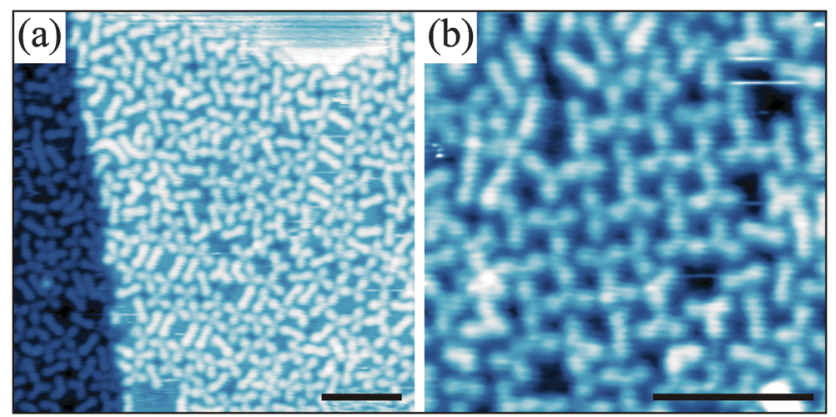

Fig. 4 Disordered Ni-1 coordination networks on $\mathrm{Ag}(001)$. Ni was deposited on $1 / \mathrm{Ag}(001)$ at $430 \mathrm{~K}$ (a) and additional deposition of 1 and annealing at $430 \mathrm{~K}$ resulted in (b). Images acquired at $300 \mathrm{~K}$. Scale bars are $5 \mathrm{~nm}$. high degree of flexibility of the individual components this results in divergent coordination assemblies with polymorphic coordination motifs that are close in energy and may render the observed disordered networks thermodynamically stable. ${ }^{7}$ No significant changes were observed upon annealing up to $460 \mathrm{~K}$, the temperature at which molecular decomposition starts. Only at low Ni concentration the L-shaped ligands are absent and ordered domains resembling locally the $\mathrm{Cu}-\mathbf{1}$ molecular domains (except for translation symmetry) ${ }^{9}$ were observed that are trapped inside larger disordered networks or molecular phases (Fig. 4b).

The disordered phase evolves from the ordered deprotonated phase of $\mathbf{1}$ after $\mathrm{Ni}$ deposition at an elevated temperature of $430 \mathrm{~K}$. As presented in more detail in the ESI, $\dagger$ the coordination motifs include predominantly the alkynyl group (90-98\%); moreover, a relatively large portion of hydrogen bonds is still found within the disordered coordination network. The dominant coordination modes incorporating single Ni atoms comprise one alkynyl and either one or two carboxylate groups. Taking into account only binding motifs in which all molecules include Ni-alkynyl coordination, three main structural units containing 3 or 4 molecules with a Ni: 1 ratio of $1: 1$ (see Fig. 5) are found. Neither of these modes can be combined to form a long-range ordered lattice on the surface.

The Ni-1 structures obtained on the Ag(001) surface are in marked contrast to the $\mathrm{Au}(111)$ surface. The Ni-alkynyl interaction is quenched on the $\mathrm{Au}(111)$ surface $^{17}$ and consequently long-range ordered Ni-1 coordination networks expressing Ni-carboxylate motifs similar to Fe-1 ones are found for the same preparation conditions. Thus, the Ni-alkynyl interaction depends strongly on the electronic hybridisation of the ligand with the surface, since the $\mathrm{Ni}$ adatoms are expected to behave very similarly on the two metal supports. ${ }^{16} \mathrm{We}$ assume that the organic molecules hybridise more strongly with the $\mathrm{Ag}(001)$ surface and that $\mathbf{1}$ on $\mathrm{Au}(111)$ represents a weakly interacting case. ${ }^{16}$ This is even more surprising because Ni-alkynyl interactions are well-known in coordination compounds. ${ }^{19}$ Furthermore, the comparison between $\mathrm{Ni}-\mathbf{1}$ and $\mathrm{Cu}-\mathbf{1}$ networks grown on the $\mathrm{Ag}(001)$ and $\mathrm{Cu}(001)$ surface, respectively, hints that the strength of the coordination bonding between the metal adatoms and the alkynyl moieties also has a dramatic effect on the overall network formation. While the $\mathrm{Cu}-\mathbf{1}$ domains exhibit long-range order presumably due to a rather weak coordination of $\mathrm{Cu}$ atoms and a

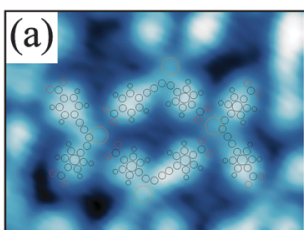

(d)

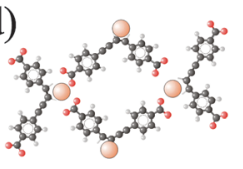

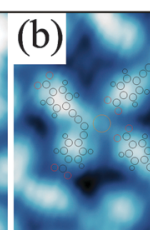

(e)

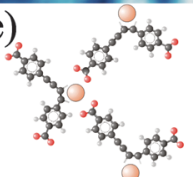

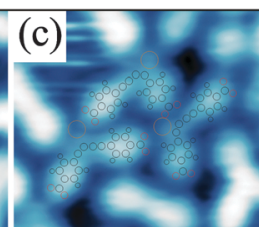

(f)

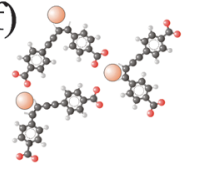

Fig. 5 (a-c) Dominant structural units observed in Ni-1 1:1 coordination networks on $\mathrm{Ag}(001)$ and $(\mathrm{d}-\mathrm{f})$ corresponding tentative models, respectively. 
symmetrically bent ligand, the strong binding of $\mathrm{Ni}$ to the butadiynyl backbone that leads to L-shaped ligands with lowered symmetry results in both the suppression of long-range order and occurrence of random networks. This demonstrates that disorder can occur in systems in which several equivalent asymmetric binding motifs are possible, where also the registry of the substrate cannot favour a structural motif.

Non-reversible coordination bond formation generally causes an increased tendency to form non-periodic assemblies. ${ }^{7,8,20}$ In addition, the Ni-alkynyl coordination gives rise to multiplicity by the emergent stereochemistry of the diverse $\mathrm{Ni}-\mathbf{1}$ conformers introducing disorder into the assembled structure that cannot be cured by post-annealing treatments. ${ }^{3,7,15}$ Thus random coordination assemblies can occur, ${ }^{7}$ for which the possible thermodynamic stability stems from the large entropy associated with the bond/ linker flexibility. ${ }^{14}$ However, to discriminate whether the observed disordered structure is kinetically trapped (glass-like) or thermodynamically stable, detailed theoretical calculations of the different binding modes are required.

In conclusion, our results demonstrate that the underlying substrate plays a crucial role in the formation of all observed coordination structures as the chemical hybridisation of the ligand activates or suppresses particular coordination motifs. The alkynyl functionality shows a high degree of flexibility in contact with the metal substrate, which allows the formation of unique network structures for each substrate. Furthermore, the coordination of $\mathrm{Ni}$ atoms with the central butadiyne group results in a strong bending of the molecule, however, the chemical coupling of $\mathbf{1}$ to the supporting surface can enable (Ni-1/Ag) or quench (Ni-1/Au) $\mathrm{Ni}$-alkynyl interaction resulting in random or ordered networks, respectively. Hence, by balancing the strength of interactions via metal adatom and substrate-controlled activation of particular functional groups a particular ligand system 1 can be switched between convergent and divergent $2 \mathrm{D}$ assemblies.

This work was supported by the Baden-Württemberg Stiftung. J. Č. acknowledges the support provided by Marie Curie IEF within the 7th FP (AdaptNano, Project No. 251930) and the European Regional Development Fund - project CEITEC (CZ.1.05/1.1.00/02.0068).

\section{Notes and references}

1 J. V. Barth, G. Constantini and K. Kern, Nature, 2005, 437, 671; L. Bartels, Nat. Chem., 2010, 2, 87; J. A. A. W. Elemans, S. Lei and S. De Feyter, Angew. Chem., Int. Ed., 2009, 48, 7298.

2 R. E. A. Kelly, M. Lukas, L. N. Kantorovich, R. Otero, W. Xu, M. Mura, E. Laegsgaard, I. Stensgaard and F. Besenbacher, J. Chem. Phys., 2008, 129, 184707.

3 A. Langner, S. L. Tait, N. Lin, C. Rajadurai, M. Ruben and K. Kern, Proc. Natl. Acad. Sci. U. S. A., 2007, 104, 17927.

4 N. A. Wasio, R. C. Quardokus, R. P. Forrest, C. S. Lent, S. A. Corcelli, J. A. Christie, K. W. Henderson and S. A. Kandel, Nature, 2014, 507, 86.

5 D. Ecija, S. Vijayaraghavan, W. Auwarter, S. Joshi, K. Seufert, C. Aurisicchio, D. Bonifazi and J. V. Barth, ACS Nano, 2012, 6, 4258.

6 R. Otero, M. Lukas, R. E. A. Kelly, W. Xu, E. Laegsgaard, I. Stensgaard, L. N. Kantorovich and F. Besenbacher, Science, 2008, 319, 312.

7 M. Marschall, J. Reichert, A. Weber-Bargioni, K. Seufert, W. Auwarter, S. Klyatskaya, G. Zoppellaro, M. Ruben and J. V. Barth, Nat. Chem., 2010, 2, 131 .

8 J. Reichert, M. Marschall, K. Seufert, D. Ecija, W. Auwärter, E. Arras, S. Klyatskaya, M. Ruben and J. V. Barth, J. Phys. Chem. C, 2013, 117, 12858

9 M. O. Blunt, J. C. Russell, M. del Carmen Gimenez-Lopez, J. P. Garrahan, X. Lin, M. Schroder, N. R. Champness and P. H. Beton, Science, 2008, 322, 10779; A. Stannard, J. C. Russell, M. O. Blunt, C. Salesiotis, M. del Carmen Gimenez-Lopez, N. Taleb, M. Schroder, N. R. Champness, J. P. Garrahan and P. H. Beton, Nat. Chem., 2012, 4, 112.

10 C. S. Kley, J. Cechal, T. Kumagai, F. Schramm, M. Ruben, S. Stepanow and K. Kern, J. Am. Chem. Soc., 2012, 134, 6072.

11 M. E. Canas-Ventura, K. Ait-Mansour, P. Ruffieux, R. Rieger, K. Mullen, H. Brune and R. Fasel, ACS Nano, 2011, 5, 457.

12 S. Whitelam, I. Tamblyn, P. H. Beton and J. P. Garrahan, Phys. Rev. Lett., 2012, 108, 035702.

13 T. K. Haxton, H. Zhou, I. Tamblyn, D. Eom, Z. Hu, J. B. Neaton, T. F. Heinz and S. Whitelam, Phys. Rev. Lett., 2013, 111, 265701.

14 S. Whitelam, I. Tamblyn, T. K. Haxton, M. B. Wieland, N. R. Champness, J. P. Garrahan and P. H. Beton, Phys. Rev. X, 2014, 4, 011044.

15 D. Nieckarz and P. Szabelski, J. Phys. Chem. C, 2013, 117, 11229.

16 N. Abdurakhmanova, T.-C. Tseng, A. Langner, C. S. Kley, V. Sessi, S. Stepanow and K. Kern, Phys. Rev. Lett., 2013, 110, 027202.

17 J. Cechal, C. S. Kley, T. Kumagai, F. Schramm, M. Ruben, S. Stepanow and K. Kern, J. Phys. Chem. C, 2013, 117, 8871.

18 B. C. Stipe, M. A. Rezaei and W. Ho, Phys. Rev. Lett., 1999, 82, 1724; X. F. Hu, C. J. Chen and J. C. Tang, Surf. Sci., 1996, 365, 319.

19 U. Rosenthal, C. Nauck, P. Arndt, S. Pulst, W. Baumann, V. V. Burlakov and H. Görls, J. Organomet. Chem., 1994, 484, 81.

20 M. Marschall, J. Reichert, K. Seufert, W. Auwarter, F. Klappenberger, A. Weber-Bargioni, S. Klyatskaya, G. Zoppellaro, A. Nefedov, T. Strunskus, C. Woll, M. Ruben and J. V. Barth, ChemPhysChem, 2010, 11, 1446. 